\title{
Characterization of Cardiac-derived Myelo-monocytoid Progenitor Cells
}

\author{
Marc-Estienne Roehrich ${ }^{1}$, Lucio Barile ${ }^{2}$, Albert Spicher ${ }^{1}$, Mauro Giacca ${ }^{3}$ and Giuseppe Vassalli ${ }^{1,2 *}$ \\ ${ }^{1}$ Department of Cardiology, Centre Hospitalier Universitaire Vaudois (CHUV), University of Lausanne, Lausanne, Switzerland \\ ${ }^{2}$ Molecular Cardiology Laboratory, Fondazione Cardiocentro Ticino, Lugano, Switzerland
}

${ }^{3}$ International Center for Genetic Engineering and Biotechnology, Trieste, Italy

\begin{abstract}
Cells migrating from heart tissue biopsies during ex vivo culture have been associated with cardiomyogenic and angiogenic potential. We have characterized cells shed by murine cardiac explants cultured in MesenCult MSC medium. A relatively uniform population with a distinguishable morphology emerged from the early cellular outgrowth. This population expressed monocyte/macrophage and hematopoietic markers $\left(\mathrm{CD} 11 \mathrm{~b}^{+}, \mathrm{CD} 14^{+}, \mathrm{CD} 45^{+}\right)$

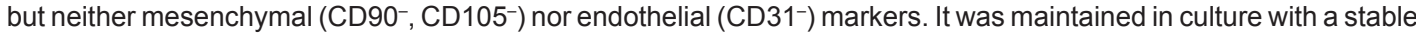
phenotype for more than 10 months. When cultured in differentiation medium, cells expressed cardiac sarcomeric a-actinin. They spontaneously formed sphere clusters ("cardiospheres"). This process was enhanced by TNFa. Spheres from neonatal mice exhibited spontaneous beating. To assess whether "contaminating" myocardial tissue fragments would explain CS beating, neonatal Z/EG transgenic mice were injected with an adeno-associated virus (AAV) vector expressing Cre-recombinase from a cardiac-specific promoter (Ncx1), resulting in the excision of a lacZ gene and activatation of the expression of the second reporter gene, enhanced green fluorescent protein (EGFP). Cardiac explants from Z/EG mice receiving AAV-9.Ncx1-CRE expressed EGFP, whereas the cellular outgrowth did not, demonstrating that the presence of cardiomyocytes in the outgrowth was not required for the generation of cardiac progenitor cells. Whether the myelo-monocytoid population originated from a retained hematologic component of explants or cardiac-resident leukocytes remains unclear. These results are in line with recent data on monocyte-derived multipotent cells (MOMC) with cardiomyogenic potential. They suggest that the cardiac-derived myelo-monocytoid cell, although not considered a classical adult progenitor cell, may have regenerative potential.
\end{abstract}

Keywords: Stem cell; Progenitor; Heart; Mouse; Monocyte

\section{Introduction}

Over the past decade, accumulating evidence has suggested that the adult mammalian heart may harbor one or more populations of progenitor cells endowed with regenerative potential [1]. Whether these cells represent adult cardiac-resident progenitor cells (CPCs) or bone marrow (BM)-derived cells homing to the heart is incompletely understood. We and others have shown that BM-derived cells can acquire stem cell properties in the damaged heart $[2,3]$. At minimum, 5 seemingly different populations of cardiac stem cells have been described [4]. Whether these populations represent distinct entities of cardiac stem cells or different developmental and/or physiological stages of a single entity of stem cell remains unclear.

For the prospective isolation of CPCs, many studies have relied upon specific antibodies that recognize cell-surface antigen markers expressed by stem cells in other tissues, and particularly by hematopoietic stem cells, such as c-kit (CD117; stem cell factor receptor) [5] and stem cell antigen-1 (Sca-1) [6]. However, no single cell surface marker definitely identifies a single entity of CPC.

Alternatively, progenitor cells have been obtained from heart tissue biopsies maintained in primary culture [7-14]. Cells shed by cardiac explants in primary culture initially form a layer of fibroblast-like cells on which numerous small round "phase-bright" cells appear with a delay of 1 to 2 weeks [11]. The cellular outgrowth from murine neonatal cardiac explants is heterogeneous and includes both hematopoietic $\left(\mathrm{CD} 45^{+}\right)$and non-hematopoietic $\left(\mathrm{CD} 45^{-}\right)$cells. The hematopoietic component of the cellular outgrowth can be minimized by perfusing the heart with heparinized saline before placing the tissue explant in culture [12]. This observation suggests that $\mathrm{CD} 45^{+}$cells in the cellular outgrowth may originate from a retained hematologic component. The cellular outgrowth from saline-perfused hearts displayed a heterogeneous immunophenotype consistent with a mixed population of CPCs (differentiation lineage-negative [ $\left.\mathrm{Lin}^{-}\right], \mathrm{c}-\mathrm{kit}^{+}$), endothelial cells $\left(\mathrm{CD} 31^{+}, \mathrm{CD} 34^{+}\right)$, and mesenchymal/stromal cells $\left(\mathrm{CD} 90^{+}\right.$, $\left.\mathrm{CD} 105^{+}\right)$, but not hematopoietic cells $\left(\mathrm{CD} 45^{-}\right)$.

Cells shed by cultured cardiac explants from rodents and humans can form multicellular sphere clusters, or "cardiospheres" (CS). CS formation is associated with an increase in progenitor cells, endothelial cells and mesenchymal cells but a decrease in hematopoietic cells $[1,12,13]$. It has been suggested that human CS may result from stem cells endowed with cardiomyogenic and angiogenic potential $[12,13]$ In animal models of myocardial infarction, CS as well as CS-derived cells injected intramyocardially enhanced blood vessel formation and functional recovery. However, a recent study challenged the view that CS from rodents are a source of stem cells with cardiomyogenic potential [14]. This study claimed that beating CS were explained by "contaminating" myocardial tissue fragments that detached from the explants as these became less cohesive after extended periods of time in culture. Removing cell aggregates with filters minimized CS beating. This issue remains controversial [14]. Here, we have isolated, expanded

${ }^{*}$ Corresponding authors: Giuseppe Vassalli, Cardiology, Department of Cardiology, Centre Hospitalier Universitaire Vaudois (CHUV), University of Lausanne, Lausanne, Switzerland, E-mail: giuseppe.vassalli@chuv.ch

Marc-Estienne Roehrich, Department of Cardiology, Centre Hospitalier Universitaire Vaudois (CHUV), University of Lausanne, Lausanne, Switzerland, E-mail: marc-estienne.roehrich@chuv.ch

Received April 30, 2012; Accepted June 22, 2012; Published June 24, 2012

Citation: Roehrich ME, Barile L, Spicher A, Giacca M, Vassalli G (2012) Characterization of Cardiac-derived Myelo-monocytoid Progenitor Cells. J Stem Cell Res Ther S9:002. doi:10.4172/2157-7633.S9-002

Copyright: (C) 2012 Roehrich ME, et al. This is an open-access article distributed under the terms of the Creative Commons Attribution License, which permits unrestricted use, distribution, and reproduction in any medium, provided the original author and source are credited. 
and characterized cells shed by cardiac explants from neonatal or adult mice in primary culture. We consistently obtained a relatively uniform cell population with a distinguishable morphology, which expressed monocyte/macrophage and hematopoietic markers, but neither endothelial nor MSC markers (with the exception of CD44). These myelo-monocytoid cells grew slowly but could be maintained in culture with a stable phenotype for more than 10 months. Cells from neonatal mice, but not those from adult mice, formed spontaneously beating CS. To assess whether "contaminating" myocardial tissue fragments could explain CS beating, we used the Z/EG double-reporter system. Cardiac-specific expression of Cre-recombinase resulted in the excision of a lacZ gene and activation of expression of the second reporter gene (enhanced green fluorescent protein; EGFP) in the heart of Z/EG transgenic mice. After Cre-recombinase gene transfer, EGFP expression was observed in $\mathrm{Z} / \mathrm{EG}$ heart tissue explants but not in the cellular outgrowth, demonstrating that the presence of cardiomyocytes in the outgrowth was not required for the generation of CPC. These findings suggest that myelo-monocytoid cells isolated from cardiac explant outgrowths may be of potential interest as a cell source for cardiac repair.

\section{Materials and Methods}

\section{Cell isolation and culture}

Hearts were collected from neonatal (1-2 days of age) or adult (810 weeks of age) male C57Bl/6 mice (Charles River Labs, France). The neonatal heart was rinsed abundantly with ice-cold heparinized saline and ventricles were cut into small pieces that were placed in Corning Costar 6-well plates (Sigma) with no extracellular matrix (ECM) protein coating. The adult heart was retrogradely perfused with ice-cold heparinized saline and ventricles were cut into small pieces that were incubated with $5 \mathrm{~mL}$ RPMI supplemented with $12.5 \mu \mathrm{L}$ Liberase II and $25 \mu \mathrm{L}$ DNAse I (both from Roche) for $30 \mathrm{~min}$ at $37^{\circ} \mathrm{C}$ (this treatment was performed because of the higher ECM content of the adult heart compared with the neonatal). Tissue pieces were placed in $5.5-\mathrm{cm}$ (Greiner) or $10 \mathrm{~cm}$ plastic dishes (Sarstedt) with no extracellular matrix (ECM) protein coating. Cardiac explants were cultured in MesenCult medium consisting of MesenCult MSC Basal Medium supplemented with serum-containing MesenCult MSC Stimulatory Supplements (both from Stem Cell Technologies). After 2 weeks, tissue explants were removed from the plates or dishes, and the cellular outgrowth was allowed to grow as a plastic-adherent monolayer. When cells reached near-confluence, they were detached from plastic with PBS-EDTA, collected, and seeded onto new plates.

\section{Flow cytometric analyses}

For flow cytometric analysis, cells were gently detached from plastic with PBS-EDTA, filtered through a $70-\mu \mathrm{m}$ filter, centrifuged, and resuspended in reagent A Leucoperm B4FO9B (AbD Serotec) for $15 \mathrm{~min}$ at RT. Then, they were washed in PBS, centrifuged, resuspended in reagent $\mathrm{B}$, incubated with primary antibody (Supplementary Table 1) for 30 min at RT, washed with PBS and, when necessary, labeled with Alexa 488 (Invitrogen; 1:25 dilution) for $30 \mathrm{~min}$ at RT. Flow cytometric analysis was performed using a FACSCalibur system (BD Bioscience) and the CellQuest software. Gates used to resolve antigen-expressing cells were set using appropriate isotype-specific control antibodies.

\section{Differentiation media}

To induce cardiac differentiation, adherent cells were grown in MesenCult medium for 2-3 months and then transferred to differentiation medium consisting of MesenCult medium supplemented with $1.3 \mu \mathrm{M}$ dexamethasone, $50 \mu \mathrm{g} / \mathrm{mL}$ ascorbic acid, and $10 \mathrm{mM}$ glycerol-2 phosphate for 14 days [15]. To induce adipogenic differentiation, cells were transferred to MesenCult medium supplemented with $1.3 \mu \mathrm{M}$ dexamethasone.

\section{Immunocytochemistry}

Immunohistochemistry was performed as previously described [15]. Briefly, cells were seeded on Lab-Tek Chamber-Slides (Nunc) and fixed with $4 \%$ paraformaldheyde (PFA). For immunostaining of sarcomeric $\alpha$-actinin, a monoclonal anti- $\alpha$-actinin sarcomeric antibody (Sigma; 1:750 dilution) labeled with Alexa 488 (Invitrogen; 1:400 dilution) was used. For immunostaining of $\alpha$-smooth muscle actin ( $\alpha$-SMA), a rabbit polyclonal anti- $\alpha$-SMA antibody (Abcam; 1:750 dilution) labeled with Alexa 594 (Invitrogen; 1:400 dilution) was used. Nuclei were stained with DAPI. CS were stained with anti-tubulin A antibody labeled with Alexa 488, and nuclei were stained with Hoechst dye (1:1000 dilution).

\section{Reverse transcription-polymerase chain reaction (RT-PCR)}

Total mRNA was extracted using the RNeasy Mini kit (Qiagen), as described [15]. The different cDNAs were generated using the Quantitect reverse transcription kit (Qiagen) on 500 ng total RNA, with a genomic DNA digestion step. The cDNA was then used for quantification using the Quantitect SYBR Green PCR Kit (Qiagen) and the RotorGene 2000 (Qiagen). Quantitative PCRs were set up in $10 \mathrm{~mL}$ reaction volume with $5 \mathrm{~mL}$ of Quantimix, $0.5 \mathrm{~mL}$ of $10 \mathrm{mM}$ forward primer, 0.5 $\mathrm{mL}$ of $10 \mathrm{mM}$ reverse primer, $3 \mathrm{~mL} \mathrm{cDNA}$, and $1 \mathrm{~mL}$ water. The polymerase was heat-activated for $15 \mathrm{~min}$ at $95^{\circ} \mathrm{C}$. The reactions were then cycled 50 times (948C, $15 \mathrm{~s} ; 508 \mathrm{C}, 30 \mathrm{~s} ; 728 \mathrm{C}, 30 \mathrm{~s}$ ) followed by a melting step. The following primers were used for SYBR Green real-time PCR: GATA4, fw: 50-ccccaatctcgatatgtttga-30, rev: 50-atggccccacaattgaca-30; cardiac $\alpha$-actinin, fw: 50-gctgctccaactgaccctgtcc-30, rev: 50 -cggcccacgatggacgggaa-30; GAPDH, fw: 50-tcaagaaggtggtgaagcag-30, rev: 50-aagtggaagagtgggagtt-30. Relative expression was calculated using the comparative DDCT method. GAPDH was used as a reference gene.

\section{CS formation}

To study CS formation, the explant outgrowth from neonatal or adult mice was cultured in MesenCult medium for 2 to 5 months. In a subset of experiments, MesenCult medium was changed to Dulbeccomodified Eagle medium supplemented with $10 \%$ fetal calf serum (DMEM-10\% FCS) to compare the two media in terms of CS formation. Moreover, the effect of cytokines and growth factors on CS formation was addressed by supplementing MesenCult medium with $5 \mathrm{ng} / \mathrm{mL}$ of tumor necrosis factor- $\alpha$ (TNF- $\alpha$ ), bone morphogenetic proteins (BMP) 2 and 4, or transforming growth factor- $\beta 1$ (TGF $\beta 1$ ), or with $10 \mathrm{ng} / \mathrm{mL}$ of vascular endothelial growth factor-165 $\left(\mathrm{VEGF}_{165}\right)$ or basic fibroblast growth factor (bFGF). In separate experiments, CS formed in MesenCult were transferred to low-serum medium consisting of 2 volumes of B27-based medium (100 mL DMEM supplemented with Glutamax, $2 \mathrm{~mL}$ B27 50x [Gibco], and $1 \mathrm{~mL}$ penicillin/streptomycin/ fungizone 100x) and 1 volume of MesenCult medium. Cell cultures were monitored for the appearance of beating CS under the microscope. Epinephrine was used to test the responsiveness of CS beating rates to an adrenergic stimulus.

\section{Experiments using $\mathrm{Z} / \mathrm{EG}$ transgenic mice}

To determine whether the cellular outgrowth from cardiac explants contained "contaminating" myocardial tissue fragments, we 
Citation: Roehrich ME, Barile L, Spicher A, Giacca M, Vassalli G (2012) Characterization of Cardiac-derived Myelo-monocytoid Progenitor Cells. J Stem Cell Res Ther S9:002. doi:10.4172/2157-7633.S9-002

Page 3 of 7

used the Z/EG double-reporter system. In Z/EG transgenic mice, Crerecombinase (CRE) activity results in the excision of a lac $Z$ reporter gene and the concomitant activation of the expression of enhanced green fluorescent protein (EGFP) as the second reporter gene [16]. Cardiac-specific expression of CRE was mediated by a serotype- 9 adeno-associated virus vector containing the Ncx1 promoter (AAV-9. Ncx1-CRE). A vector containing a cytomegalovirus immediate-early promoter (AAV-9.CMV-CRE) was used as a control. AAV vectors were generated and characterized as described [17]. Vector stock titers ranged from $1 \times 10^{12}$ to $1 \times 10^{13}$ viral genome copies per milliliter. AAV vectors were injected intraperitoneally to neonatal mice anesthetized with $4 \%$ isoflurane. Green fluorescence in cardiac explants and cellular outgrowths was assessed by fluorescence microscopy.

\section{Statistical analysis}

The non-parametric Mann-Whitney-U test was used for comparisons between two groups. $P<0.05$ was considered to be statistically significant.

\section{Results}

\section{Cell isolation and culture}

Cultured cardiac explants from neonatal or adult mice gave rise to cellular outgrowths becoming visible after 3-4 days. The early outgrowth was comprised of spindle-shaped cells and round, highly refringent (phase-bright) cells (Figure 1). Tissue explants were removed from culture after 2 weeks. After 4-6 weeks, cells from neonatal or adult mice acquired a relatively uniform, distinguishable morphology characterized by one or multiple, thin cytoplasmic processes (the compared the shape of cells with a single process to that of gingko biloba leaves; Figure 2). Cells grew slowly but could be maintained in culture for more than 10 months with a stable morphology and immunophenotype (see below).

\section{Flow cytometric analyses}

Flow cytometric analyses of cell surface antigens were performed on cardiac outgrowth-derived cells from neonatal mice $(n=5)$ after 2 to 5 months in culture, as well as from adult mice $(n=3)$ after 2 to 9 months in culture. Cells from neonatal or adult mice displayed similar cell-surface marker profiles (Figure 3 ). These were characterized by expression of monocyte/macrophage and hematopoietic markers
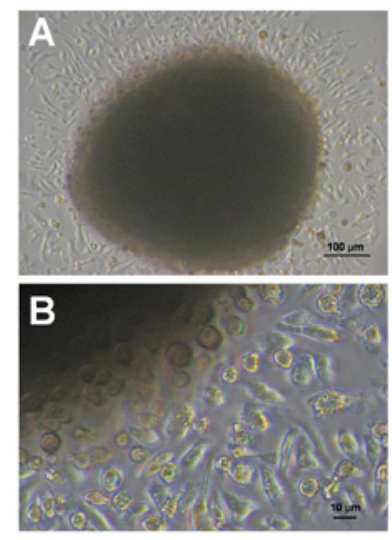

Figure 1: Phase photomicrographs of a cardiac explant outgrowth. A. Low magnification view. B. High magnification view showing a heterogeneous cell morphology including both spindle-shaped and round cells.

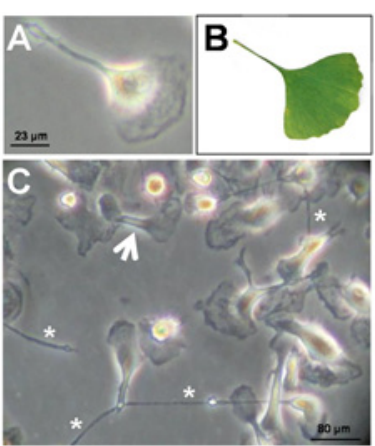

Figure 2: Phase photomicrographs of cardiac outgrowth-derived cells from neonatal mice. A. High-magnification view showing the characteristic cell morphology with a broad cytoplasmic extension at one pole of the cell and a thin cytoplasmic process at the opposite pole. B. Gingko biloba leaf, to which the cell shape has been compared. C. Cells after 50 days in culture (arrow, elongated cell process; asterisks, cytoplasmic filaments apparently establishing intercellular contacts).

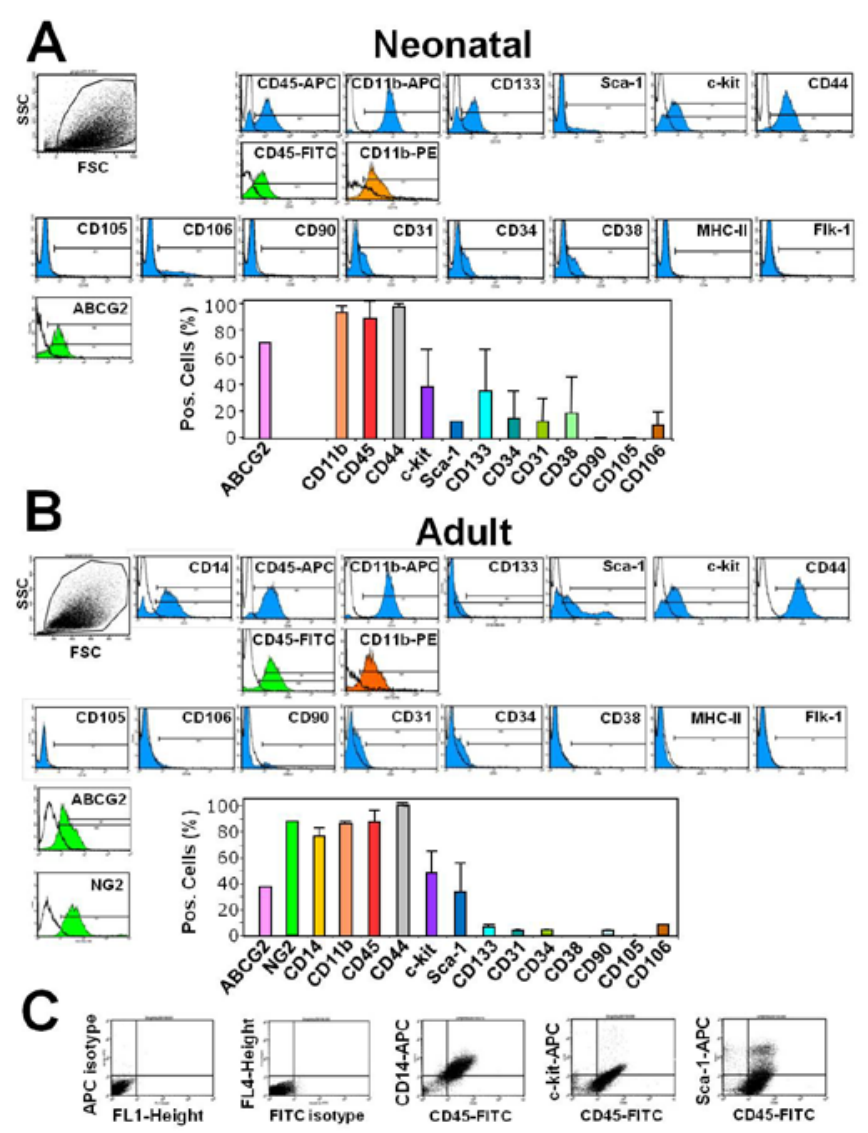

Figure 3: Flow cytometric analyses of cell-surface marker profiles of cardiac outgrowth-derived cells. A. Cells from neonatal mice. Flow cytometric plots from a representative analysis 5 months after cell isolation (two different antibodies were used for CD45 and CD11b analysis); bar histogram showing mean values $( \pm \mathrm{SD})$ from 5 independent analyses (2-5 months after cell isolation), excepted for ABCG2 and Sca-1 (1 analysis). B. Cells from adult mice. Flow cytometric plots from a representative analysis 9 months after cell isolation; bar histogram showing mean values $( \pm S D)$ from 3 independent analyses (2-9 months after cell isolation), excepted for ABCG2, NG2 and CD106 (1 analysis). C. Double-label analyses of CD45, CD14, c-kit, and Sca-1 expression. 
(CD11b, CD14, CD45), CD44 (considered a MSC and endothelial progenitor cell [EPC] marker), and NG2 proteoglycan (a marker of pericytes/perivascular cells and mesoangioblasts). Regarding stem cellassociated markers, ATP-binding cassette G2 (ABCG2) was expressed by a significant subpopulation, CD133 was expressed by a subpopulation from neonatal but not adult mice, and Sca-1 was expressed by a larger subpopulation from adult compared with neonatal mice. The specificity of Sca-1 detection was tested using cells from Sca-1-deficient mice (data not shown). CD90 and CD105 (MSC markers), CD31 (an endothelial marker), CD34 (a hematopoietic and EPC marker), CD3e (T-cell receptor complex subunit), CD19, CD38 and CD45R (B-cell markers), CD49a, CD106 (vascular cell adhesion molecule-1; VCAM-1), Flk-1 and major histocompatibility complex (MHC) class II molecules were essentially undetectable. Double-label analyses showed that c-kit ${ }^{+}$ cells and a majority of Sca- $1^{+}$cells were $\mathrm{CD} 45^{+}$. To assess changes of the cell-surface marker profile over time, the same cell population from a neonatal heart was analyzed by flow cytometry after 2 and 5 months (data are shown in Figure 4C and Figure 3A, respectively). Occasionally, cells lost their original morphology, enlarged, and ceased growth. To assess changes in cell-surface markers associated with morphological changes, we analyzed two subpopulations derived from the same population after 2 months inculture, the first one having partially lost the original morphology, and the second one having maintained it (Figure $4 \mathrm{~A}$ and $4 \mathrm{~B}$, respectively). The subpopulation with altered morphology showed decreased percentages of $\mathrm{CD} 11 \mathrm{~b}^{+}$and $\mathrm{CD}^{2} 5^{+}$cells but increased percentages of $\mathrm{CD} 106^{+}$cells compared with the subpopulation with unchanged morphology. These changes were paralled by increased mRNA expression of GATA- 4 and sarcomeric $a$-actinin. These data suggest a shift from a hematopoietic to a nonhematopoietic phenotype.

\section{Cell differentiation potential}

Cardiac outgrowth-derived cells cultured in cardiac differentiation medium stained positive for sarcomeric $\alpha$-actinin (Figure 5), which appeared to be organized in microfibrils. A minority of cells stained positive for a -SMA. Cells cultured in Mesencult supplemented with $1.3 \mu \mathrm{M}$ dexamethasone enlarged and acquired intracellular droplets indicative of adipogogenic differentiation.

\section{CS formation}

Plastic-adherent outgrowth-derived cells from neonatal or adult mice spontaneously formed CS after 2 to 5 months in culture (Figure 6). Neither low-adherence plates nor specific CS growth media were needed for CS formation. Mesencult medium was more efficient than DMEM-10\% FCS in this regard. The effect of cytokines or growth factors, supplemented to Mesencult medium, on CS formation was evaluated. TNF- $\alpha$ significantly increased the number of CS. CS from neonatal, but not adult, mice occasionally exhibited spontaneous beating activity in MesenCult medium. Virtually all CS from neonatal mice exhibited spontaneous beating in low-serum B27-based medium (see: Supplementary electronic file S1). Beating activity lasted for several days to a few weeks. Epinephrine increased beating rates up to $\approx 180 \mathrm{bpm}$.

\section{Cell tracing using the $\mathrm{Z} / \mathrm{EG}$ reporter system}

To assess whether "contaminating" myocardial tissue fragments were responsible for CS beating, we used the double-reporter Z/EG system. Neonatal Z/EG cardiomyocytes transduced with AAV-9. Ncx1-CRE or AAV-9.CMV-CRE in vitro were irreversibly labeled with EGFP (Figure 7). Neonatal cardiac fibroblasts transduced with
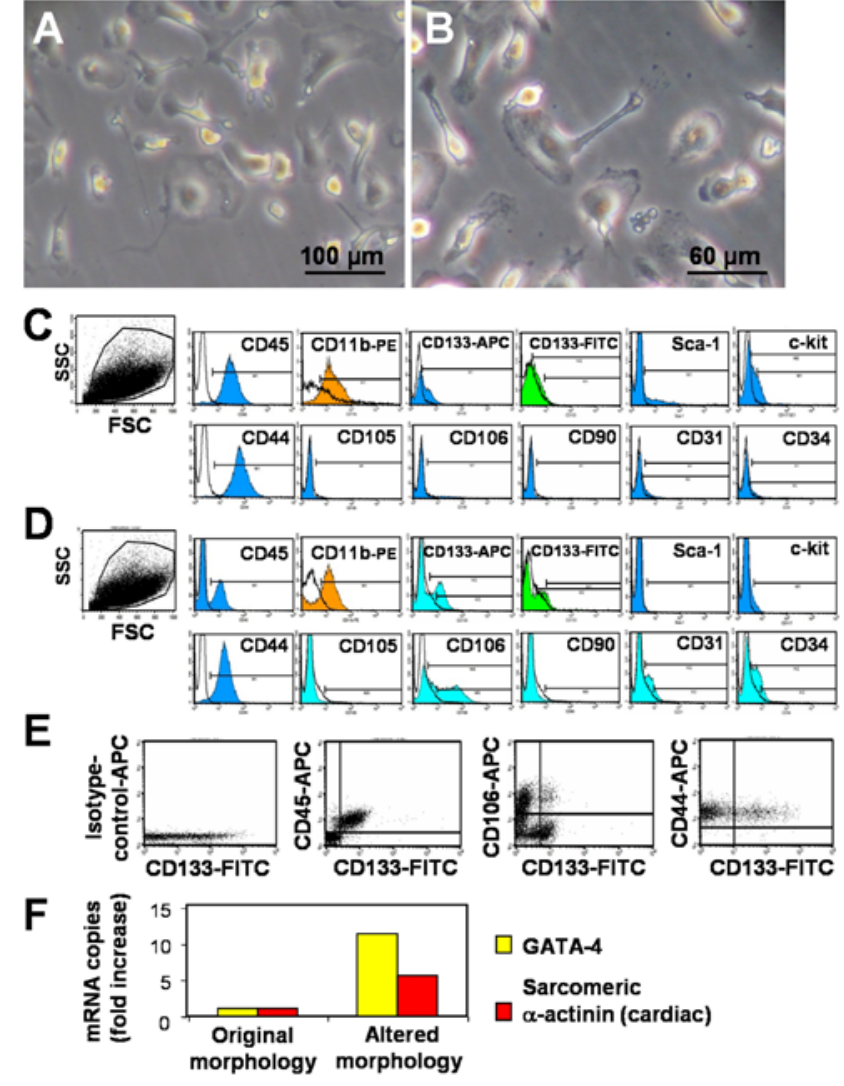

Figure 4: Comparison of two subpopulations derived from the same cardiac outgrowth population from neonatal mice. After 2 months in culture, one subpopulation partially lost the original morphology $(A)$, whereas the other maintained it (B). Flow cytometric analyses of cell-surface markers for the subpopulations with conserved (C) or altered (D) morphology (for comparison, flow cytometric data of the same population shown in panel $C$ for the 2-month time point are shown in Figure $3 \mathrm{~A}$ for the 5-month time point). (E) Double-label flow cytometric analyses of CD133, CD45, CD106 and CD44 expression in the subpopulation with conserved morphology. (F) qRT-PCR analysis of mRNA expression of GATA-4 and cardiac a-actinin (data are fold-increases in mRNA copy numbers for the subpopulation with altered morphology compared with that with conserved morphology).

AAV-9.CMV-CRE, but not those transduced with AAV-9. Ncx1-CRE, were similarly labeled. These data demonstrated cardiac-specific CRE expression using the $\mathrm{Ncx} 1$ promoter-containing vector. After 2 weeks in culture, heart tissue explants from neonatal Z/EG mice previously injected with AAV-9.Ncx1-CRE exhibited green fluorescence, whereas the cellular outgrowth did not, demonstrating lack of cardiomyocyte in the outgrowth.

\section{Discussion}

We have obtained a myelo-monocytoid cell population by outgrowth from murine cardiac explants maintained in primary tissue culture. This population exhibited a distinguishable morphology and could be maintained in culture for more than 10 months. The vast majority $(\approx 80-90 \%)$ of cells expressed the leukocyte common antigen CD45, the monocyte/macrophage markers CD11b and CD14 [1820], CD44 (generally considered a mesenchymal and EPC marker, but not expressed by primary BM MSC and progenitor cells [21]), or the pericyte/perivascular cell marker NG2 [9,22,23]. Subpopulations expressed the stem cell-associated markers ABCG2, c-kit, and CD133 
(the latter in cells from neonatal mice only). A small minority of cells expressed the endothelial marker CD31. Expression of the MSC markers CD90 and CD105 [24] was barely detectable. This cell-surface marker profile pointed to a myelo-monocytoid phenotype.

Monocyte-derived cells include macrophages, fibrocytes, dendritic cells, osteoclasts, and adipocytes [18-20]. Lack of expression of CD105 by the isolated population suggested a monocytic phenotype, rather than a macrophage or fibrocytic [19]. Expression of NG2 proteoglycan has been observed on perivascular cells including pericytes and cardiac mesoangioblasts. The latter displayed cardiomyogenic potential [9]. Moreover, human MSC in multiple organs were shown to express NG2 in vivo, thereby suggesting a perivascular origin of these cells [23]. In analogy to cardiac mesoangioblasts [9], the population isolated in the present study expressed NG2, CD44 and, in part, c-kit. On the other hand, it clearly differed from mesoangioblasts with respect to CD45, CD31, and CD34 expression.

Expression of CD44 by the isolated population may seem at odds with that of hematopoietic and monocytic markers. It should be noted, however, that blood monocyte-derived mesenchymal progenitors (MOMP) with mixed phenotypic features of monocytes, MSC, and endothelial cells have been described [25]. Moreover, MSC from BM and other tissues have been shown to express CD45 under certain conditions [26-29]. In some cases, CD45 expression by MSC was dramatically downregulated in culture [26,27]. Collectively, these data demonstrate the existence of $\mathrm{CD}_{4} 5^{+}$cells expressing mesenchymal markers, while also suggesting that certain $\mathrm{CD} 45^{-}$populations expressing such markers may have originally been $\mathrm{CD} 45^{+}$.

The myelo-monocytoid phenotype of the isolated population conflicted with published data. It should be emphasized that we used an unconventional protocol based on Mesencult medium, whereas Messina et al. [7] used an Iscove's Modified Dulbecco's Medium [IMDM]-based medium supplemented with 10\% FCS. It has been shown that the composition of the culture medium alter the phenotype of cardiac outgrowth-derived cells [12]. Several groups independently reported that human cardiac outgrowths expressed MSC markers $\left(\mathrm{CD} 90^{+}, \mathrm{CD} 105^{+}\right)$but not hematopoietic markers $\left(\mathrm{CD} 45^{-}, \mathrm{CD} 34^{-}\right.$,
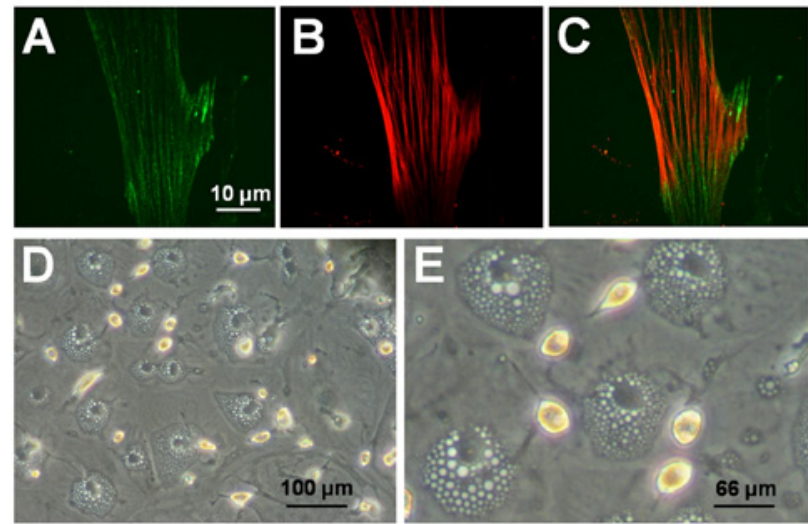

Figure 5: Cardiac outgrowth-derived cells cultured in Mesencult for 2-3 months were transferred to cardiac or adipogenic differentiation media for 14 days. A Immunostaining for sarcomeric $\alpha$-actinin with Alexa 488 (green). Nuclei were stained with DAPI (blue). B. Immunostaining for a-SMA with Alexa 594 (red) and DAPI (blue). C. Merge. D/E. Phase photomicrographs of cardiac-derived cells showing adipogenic differentiation, as evidenced by intracytoplasmic fat droplets (low and high magnification views, respectively).
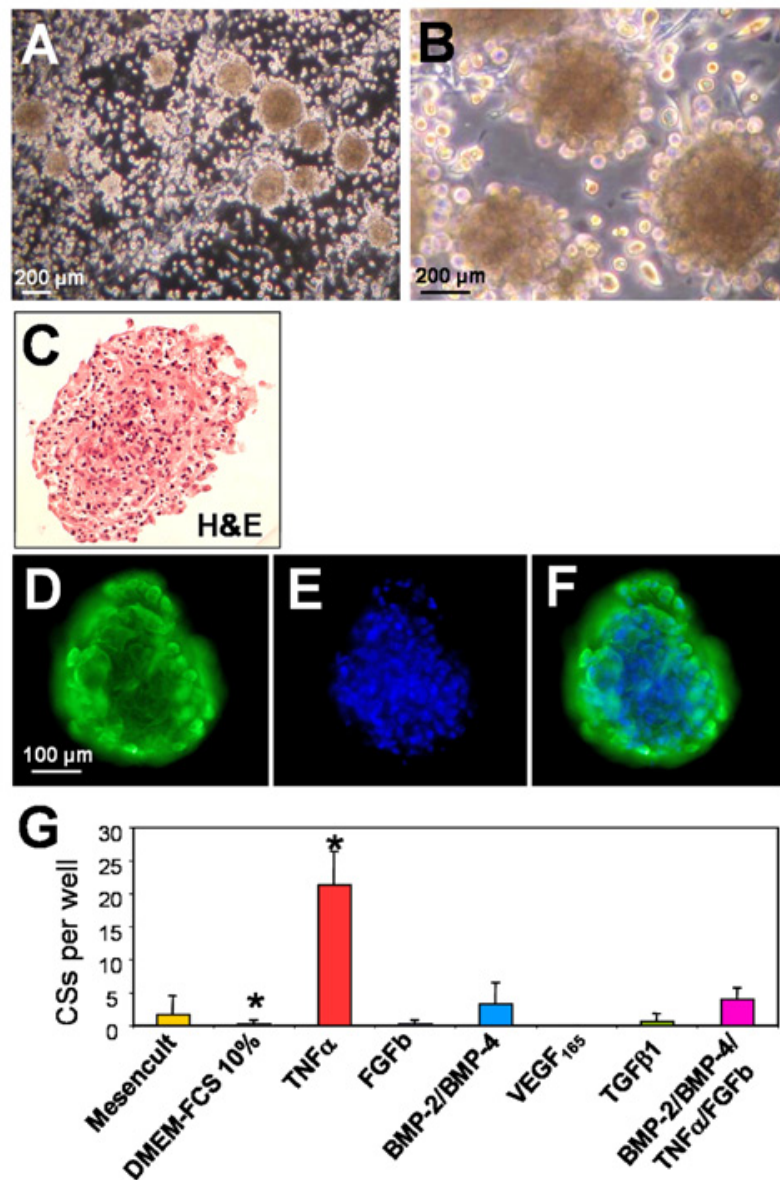

Figure 6: CS formation by cardiac outgrowth-derived cells from adult mice. A/B Photomicrographs of CS (low and high magnification views, respectively). C H\&E staining of a CS section showing nuclei in the superficial layers as well as in the core. D. Immunostaining for tubulin A (green). E. Nuclear staining with Hoechst dye (nuclei). F. Merge. G. Effects of Mesencult medium supplementation with cytokines or growth factors on CS formation; DMEM-10\% FCS was also tested for comparison (data are CS counts per well; ${ }^{*} p<0.05$ vs. Mesencult).

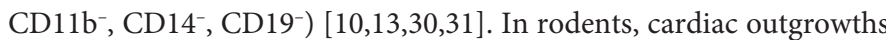
displayed a more heterogeneous phenotype. Andersen et al. [14] reported that rat cardiac outgrowths included fibroblasts, endothelial cells, EPCs, CD45- CS-forming cells, and CD45 ${ }^{+}$hematopoietic cells. Davis et al. [12] showed that retrograde perfusion of rat hearts with heparinized saline minimized the number of $\mathrm{CD} 45^{+}$cells in the cellular outgrowth. This was comprised of putative CPCs $\left(\mathrm{c}-\mathrm{kit}^{+}\right)$, endothelial cells and EPCs $\left(\mathrm{CD} 31^{+}, \mathrm{CD} 34^{+}\right)$, and mesenchymal cells $\left(\mathrm{CD} 90^{+}\right)$. In the present study, perfusion of adult hearts with heparinized saline did not prevent the isolation of $\mathrm{CD} 45^{+}$cells. However, this observation does not rule out an origin of these cells from the retained hematologic component of cardiac explants. Incidentally, it should be mentioned that we were not able to isolate a similar cell population from mouse $\mathrm{BM}$ using the same culture conditions (data not shown). An alternate hypothesis is that the isolated population originated from cardiacresident leukocytes. In this regard, it has recently been shown that the unstressed mouse heart harbors $2.3 \times 10^{3}$ resident leukocytes/mg of tissue (as measured after heart perfusion according to Langendorff with an oxygenated medium for 5 minutes), the most prominent fraction being antigen-presenting mononuclear cells $\left(\mathrm{CD} 11 \mathrm{~b}^{+}, \mathrm{CD} 11 \mathrm{c}^{+}, \mathrm{F} 4 / 80^{+}\right.$ and $\mathrm{MHC}-\mathrm{II}^{+}$) followed by B-cells, monocytes, and T-cells [32]. 
A
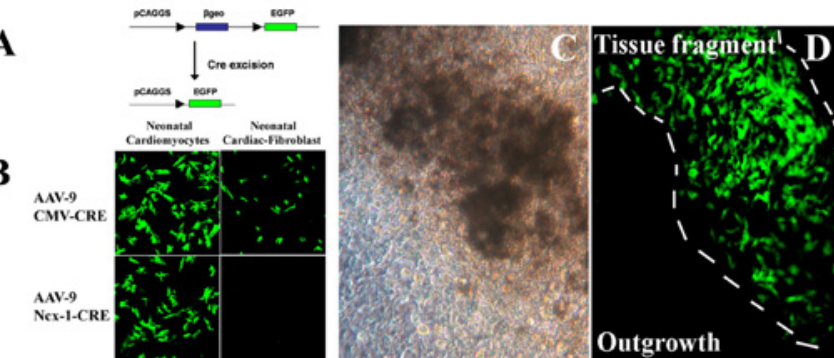

Figure 7: Primary tissue culture from a neonatal Z/EG mouse heart. Upper left panel: Schematics of the Z/EG double reporter (lacZ, EGFP) system. Lower left panel: Tissue specificity of EGFP expression using AAV-9.Ncx1-CRE as compared with AAV-9.CMV-CRE. EGFP expression using the first vector was restricted to cardiomyocytes. Middle panel: Photomicrograph of Z/EG heart tissue explant and cell outgrowth. Right panel: Corresponding fluorescence photomicrograph (the boundaries of the tissue explant are indicated by a dashed line). Green fluorescence is detectable within the explant but not in the outgrowth.

Regardless of whether the isolated population originated from blood cells trapped in the intravascular space of the explant or from cardiac-resident leukocytes, the high proportion of $\mathrm{CD} 45^{+}$and $\mathrm{CD} 11 \mathrm{~b}^{+}$ cells in the cultured population suggested an advantage for these cells in terms of survival or growth compared with other cells in the early outgrowth. Alternatively, these myelo-monocytoid cells might have resulted from the differentiation of progenitor cells. In either case, this outcome was unanticipated since MesenCult has been optimized for growth of MSC. Indeed, we previously showed that mouse cardiac MSC ( $\left.\mathrm{Lin}^{-}, \mathrm{Sca}-1^{+}, \mathrm{CD} 90^{+}, \mathrm{CD} 105^{+}, \mathrm{CD} 45^{-}, \mathrm{CD} 31^{-}\right)$could be expanded for more than 25 passages in MesenCult medium with a preserved immunophenotype [15].

Cardiac-derived myelo-monocytoid cells appeared to have cardiomyogenic potential. When cultured in differentiation medium, they stained positive for sarcomeric a-actinin. When cultured in MesenCult medium, they occasionally gave rise to freefloating multicellular sphere clusters. This process was enhanced by TNF $a$. Sphere clusters were first described with neural stem cells ("neurospheres") [33]. Spheres have been considered - or named, at least - a feature of stemness. It should be noter, however, that spheres may result from either cell proliferation or aggregation. Non-clonal spheres increase with increasing cell density in the culture plate, and therefore clonal sphere-formation assays need to be performed at very low cell densities, and ideally at the single-cell level [34]. In the present study, sphere formation was observed at relatively high cell densities, a possible hint to non-clonality. Previous studies indicated that human clonal CS resulted from stem cells with cardiomyogenic and angiogenic potential $[12,13]$. However, recent data in rodents questioned this notion [14]. Again, it is important to note methodological differences between our study and the original report by Messina et al. [7]. To induce CS formation, these investigators placed the cells in suspension culture in poly-D-lysine-coated plates in a base medium supplemented with bFGF, EGF, cardiotrophin-1, thrombin, and B27 as a serum substitute. In contrast, we observed CS formation from plastic-adherent cells (as opposed to suspension cultures) in MesenCult medium (as opposed to CS-forming medium).

The most obvious evidence of cardiomyogenic differentiation was the demonstration of beating. We occasionally observed spontaneous CS beating from neonatal mice in MesenCult medium. Virtually all spheres placed in a low-serum, B27-based medium showed spontaneous beating. Beating rates were responsive to an adrenergic stimulus, a feature of electrophysiologically mature cardiomyocytes.

Andersen et al. [14] recently suggested that CS beating from neonatal rodents might be explained by "contaminating" myocardial tissue fragments in the cellular outgrowth, especially when cardiac explants were not removed from culture plates. In the present study, tissue explants were removed from cultures after 2 weeks. In MesenCult medium, beating activity appeared 3 to 6 months after cell isolation. It seems unlikely that "contaminating" myocardial fragments would resume beating after such a long interval. To directly address this question, we used the Z/EG double-reporter system. Cardiac explants from neonatal Z/EG mice injected with the AAV-9.Ncx1CRE vector in vivo exhibited green fluorescence, whereas the cellular outgrowth did not. This observation demonstrated that the presence of cardiomyocytes in the cellular outgrowth was not required for the generation of CPC.

The theoretical possibility that cardiac-derived myelo-monocytoid cells originated from cardiac-resident MSC seems unlikely. Freisinger et al. [35] previously showed that clonally isolated, adipose tissueresident $\mathrm{MSC}\left(\mathrm{CD} 0^{+}, \mathrm{CD} 105^{+}, \mathrm{CD} 44^{+}, \mathrm{CD}^{-}, \mathrm{CD} 11 \mathrm{~b}^{-}, \mathrm{CD} 34^{-}, \mathrm{CD} 45^{-}\right.$, $\mathrm{CD}^{-} 8^{-}$) cultured in various differentiation media (IL- $1 \beta$, IL-3, M-CSF and MTG) differentiated into cells expressing early hematopoietic (c-kit) and monocyte/macrophage markers (CD11b). However, we did not use these differentiation media in the present study. Zhao et al. [36] described a subpopulation of adult pluripotent stem cells (PSC) from human peripheral blood monocytes, which in appearance resembled fibroblasts, expanded in the presence of M-CSF, expressed monocytic and hematopoietic markers (CD45, CD34, CD14), and could be induced to differentiate into various cell types in the presence of appropriate growth factors. Kuwana et al. [25,37] described human blood monocytederived mesenchymal progenitors (MOMP) as well as monocytederived multipotent cells (MOMC) expressing CD45, CD34, CD14 and type-I collagen. These cells showed a fibroblast-like morphology and mixed phenotypic features of monocytes, endothelial cells, and MSC. They contained progenitors with the capacity to differentiate into bone, cartilage, fat, skeletal and cardiac muscle, neuron, and endothelium [25,37-39]. Romagnani et al. [40] described clonogenic, multipotent, circulating $\mathrm{CD} 14^{+} \mathrm{CD} 34^{\text {low }}$ cells that proliferated in response to stem cell growth factors. Our data on myelo-monocytoid cells obtained by outgrowth from from cardiac explants are in line with these reports on multipotent monocytes. These findings suggest that the monocyte, traditionally viewed as a committed precursor for macrophages and dendritic cells, may have a larger differentiation potential. Further studies are warranted to compare the regenerative potential of cardiacderived myelo-monocytoid cells with that of other CPC populations in animal models of heart injury.

\section{Acknowledgement}

Support by the Swiss Heart Foundation, the Fondation Vaudoise de Cardiologie (Lausanne), and the Cecilia Augusta Foundation (Lugano) is gratefully acknowledged.

\section{References}

1. Barile L, Messina E, Giacomello A, Marbán E (2007) Endogenous cardiac stem cells. Prog Cardiovasc Dis 50: 31-48.

2. Barile L, Cerisoli F, Frati G, Gaetani R, Chimenti I, et al. (2011) Bone marrowderived cells can acquire cardiac stem cells properties in damaged heart. J Cell Mol Med 15: 63-71.

3. Fazel SS, Chen L, Angoulvant D, Li SH, Weisel RD, et al. (2008) Activation of c-kit is necessary for mobilization of reparative bone marrow progenitor cells in response to cardiac injury. FASEB J 22: 930-940. 
Citation: Roehrich ME, Barile L, Spicher A, Giacca M, Vassalli G (2012) Characterization of Cardiac-derived Myelo-monocytoid Progenitor Cells. J Stem Cell Res Ther S9:002. doi:10.4172/2157-7633.S9-002

Page 7 of 7

4. Ellison GM, Galuppo V, Vicinanza C, Aquila I, Waring CD et al. (2010) Cardiac stem and progenitor cell identification: different markers for the same cell?. Front Biosci (Schol Ed) 2: 641-652.

5. Beltrami AP, Barlucchi L, Torella D, Baker M, Limana F, et al. (2003) Adult cardiac stem cells are multipotent and support myocardial regeneration. Cell 114: $763-776$.

6. Tateishi K, Ashihara E, Takehara N, Nomura T, Honsho S, et al. (2007) Clonally amplified cardiac stem cells are regulated by Sca-1 signaling for efficient cardiovascular regeneration. J Cell Sci 120: 1791-1800.

7. Messina E, De Angelis L, Frati G, Morrone S, Chimenti S, et al. (2004) Isolation and expansion of adult cardiac stem cells from human and murine heart. Circ Res 95: 911-921.

8. Steele A, Jones OY, Gok F, Marikar Y, Steele P, et al. (2005) Stem-like cells traffic from heart ex vivo, expand in vitro, and can be transplanted in vivo. $J$ Heart Lung Transplant 24: 1930-1939.

9. Galvez BG, Sampaolesi M, Barbuti A, Crespi A, Covarello D, et al. (2008) Cardiac mesoangioblasts are committed, self-renewable progenitors, associated with small vessels of juvenile mouse ventricle. Cell Death Differ 15: $1417-1428$

10. Bearzi C, Rota M, Hosoda T, Tillmanns J, Nascimbene A, et al. (2007) Human cardiac stem cells. Proc Natl Acad Sci USA 104: 14068-14073.

11. Shenje LT, Field LJ, Pritchard CA, Guerin CJ, Rubart M, et al. (2008) Lineage tracing of cardiac explant derived cells. PLoS One 3: e1929.

12. Davis DR, Zhang Y, Smith RR, Cheng K, Terrovitis J, et al. (2009) Validation of the cardiosphere method to culture cardiac progenitor cells from myocardial tissue. PLoS one 4: e7195.

13. Smith RR, Barile L, Cho HC, Leppo MK, Hare JM, et al. (2007) Regenerative potential of cardiosphere-derived cells expanded from percutaneous endomyocardial biopsy specimens. Circulation 115: 896-908.

14. Andersen DC, Andersen P, Schneider M, Jensen HB, Sheikh SP (2009) Murine "cardiospheres" are not a source of stem cells with cardiomyogenic potential. Stem Cells 27: 1571-1581.

15. Meinhardt A, Spicher A, Roehrich ME, Glauche I, Vogt P, et al. (2011) Immunohistochemical and flow cytometric analysis of long-term label-retaining cells in the adult heart. Stem Cells Dev 20: 211-222.

16. Novak A, Guo C, Yang W, Nagy A, Lobe CG (2000) Z/EG, a double reporter mouse line that expresses enhanced green fluorescent protein upon Cremediated excision. Genesis 28: 147-155.

17. Zacchigna S, Tasciotti E, Kusmic C, Arsic N, Sorace OC, et al. (2007) In vivo imaging shows abnormal function of vascular endothelial growth factor-induced vasculature. Hum Gene Ther 18: 515-524.

18. Gorczyca W, Sun ZY, Cronin W, Li X, Mau S, et al. (2011) Immunophenotypic pattern of myeloid populations by flow cytometry analysis. Methods Cell Bio 103: 221-266.

19. Pilling D, Fan T, Huang D, Kaul B, Gomer RH (2009) Identification of markers that distinguish monocyte-derived fibrocytes from monocytes, macrophages, and fibroblasts. PLoS one 4: e7475.

20. Curnow SJ, Fairclough M, Schmutz C, Kissane S, Denniston AK, et al. (2010) Distinct types of fibrocyte can differentiate from mononuclear cells in the presence and absence of serum. PLoS one 5: e9730.

21. Qian H, Le Blanc K, Sigvardsson M (2012) Primary mesenchymal stem and progenitor cells from bone marrow lack expression of CD44. J Biol Chem.

22. Maier CL, Shepherd BR, Yi T, Pober JS (2010) Explant outgrowth, propagation and characterization of human pericytes. Microcirculation 17: 367-380.

23. Crisan M, Yap S, Casteilla L, Chen CW, Corselli M, et al. (2008) A perivascular origin for mesenchymal stem cells in multiple human organs. Cell Stem Cell 3: 301-313.

24. Dominici M, Le Blanc K, Mueller I, Slaper-Cortenbach I, Marini F, et al. (2006) Minimal criteria for defining multipotent mesenchymal stromal cells. The
International Society for Cellular Therapy position statement. Cytotherapy 8 : 315-317.

25. Kuwana M, Okazaki Y, Kodama H, Izumi K, Yasuoka H, et al. (2003) Human circulating CD14-monocytes as a source of progenitors that exhibit mesenchymal cell differentiation. J Leukoc Biol 74: 833-845.

26. Kaiser S, Hackanson B, Follo M, Mehlhorn A, Geiger K, et al. (2007) BM cells giving rise to MSC in culture have a heterogeneous CD34 and CD45 phenotype. Cytotherapy 9: 439-450.

27. Deschaseaux F, Gindraux F, Saadi R, Obert L, Chalmers D, et al. (2003) Direct selection of human bone marrow mesenchymal stem cells using an anti-CD49a antibody reveals their CD45med, low phenotype. Br J Haematol 122: 506-517.

28. Koide Y, Morikawa S, Mabuchi Y, Muguruma Y, Hiratsu E, et al. (2007) Two distinct stem cell lineages in murine bone marrow. Stem Cells 25: 1213-1221.

29. Rogers I, Yamanaka N, Bielecki R, Wong CJ, Chua S, et al. (2007) Identification and analysis of in vitro cultured CD45-positive cells capable of multi-lineage differentiation. Exp Cell Res 313: 1839-1852.

30. Mishra R, Vijayan K, Colletti EJ, Harrington DA, Matthiesen TS, et al. (2011) Characterization and functionality of cardiac progenitor cells in congenital heart patients. Circulation 123: 364-373.

31. Chen L, Ashraf M, Wang Y, Zhou M, Zhang J, et al. (2012) The role of Notch1 activation in cardiosphere derived cell differentiation. Stem Cells Dev.

32. Bönner F, Borg N, Burghoff S, Schrader J (2012) Resident cardiac immune cells and expression of the ectonucleotidase enzymes CD39 and CD73 after ischemic injury. PLoS one 7: e34730

33. Reynolds BA, Weiss S (1992) Generation of neurons and astrocytes from isolated cells of the adult mammalian central nervous system. Science 255 1707-1710.

34. Pastrana E, Silva-Vargas V, Doetsch F (2011) Eyes wide open: A critical review of sphere formation as an assay for stem cells. Cell Stem Cell 8: 486-498.

35. Freisinger E, Cramer C, Xia X, Murthy SN, Slakey DP, et al. (2010) Characterization of hematopoietic potential of mesenchymal stem cells. J Cell Physiol 225: 888-897.

36. Zhao Y, Glesne D, Huberman E (2003) A human peripheral blood monocytederived subset acts as pluripotent stem cells. Proc Natl Acad Sci USA 100 2426-2431.

37. Seta N, Kuwana M (2007) Human circulating monocytes as multipotentia progenitors. Keio J Med 56: 41-47.

38. Kodama H, Inoue $T$, Watanabe R, Yasutomi D, Kawakami $Y$, et al. (2006) Neurogenic potential of progenitors derived from human circulating CD14+ monocytes. Immunol Cell Biol 84: 209-217.

39. Kodama $\mathrm{H}$, Inoue $\mathrm{T}$, Watanabe $\mathrm{R}$, Yasuoka $\mathrm{H}$, Kawakami $\mathrm{Y}$, et al. (2005) Cardiomyogenic potential of mesenchymal progenitors derived from human circulating CD14+ monocytes. Stem Cells Dev 14: 676-686.

40. Romagnani $\mathrm{P}$, Annunziato F, Liotta F, Lazzeri E, Mazzinghi B, et al. (2005) CD14+CD34low cells with stem cell phenotypic and functional features are the major source of circulating endothelial progenitors. Circ Res 97: 314-322. 